\title{
What We Need to Do to Improve Our Immune System Despite the Use of Vaccination for COVID-19?
}

\author{
Huang Wei Ling* \\ Infectious Diseases, General Practice, Enteral and Parenteral Nutrition Doctor, Acupuncture and Pain Management specialist. Medical Acupuncture \\ and Pain Management Clinic, Franca, São Paulo, Brazil.
}

Corresponding Author: Huang Wei Ling, Infectious Diseases, General Practice, Enteral and Parenteral Nutrition Doctor, Acupuncture and Pain Management specialist. Medical Acupuncture and Pain Management Clinic, Franca, São Paulo, Brazil.

\section{Received Date: 12 August 2021 | Accepted Date: 28 September 2021 | Published Date: 07 October 2021}

Citation: Huang W Ling. (2021) What We Need to Do to Improve Our Immune System Despite the Use of Vaccination for COVID-19?. Biomedical Research and Clinical Reviews. 5(1); DOI: 10.31579/2692-9406/081

Copyright: () 2021 Huang Wei Ling, This is an open-access article distributed under the terms of the Creative Commons Attribution License, which permits unrestricted use, distribution, and reproduction in any medium, provided the original author and source are credited.

\begin{abstract}
:
In our daily practice, we have heard more and more frequent reports of people infected with SARS-CoV2, even having received one or two doses of vaccine for COVID-19. In the article written by Angel et al. (2021) entitled Association Between Vaccination with BNT162b2 and Incidence of Symptomatic and Asymptomatic SARS-CoV-2 Infections Among Health Care Workers, the authors concluded that the effectiveness of the vaccine in symptomatic SARS-CoV-2 infection reduced the incidence of this infection if you compared with unvaccinated health care worker. But the incidence was not reduced in the asymptomatic health care workers, where they found that vaccinated people were more likely to have SARS-CoV-2 if compared to unvaccinated people.
\end{abstract}

Keywords: immune system; covid-19; vaccination; immunity; asymptomatic sars-cov-2

\section{Introduction}

In our daily practice, we have heard more and more frequent reports of people infected with SARS-CoV-2, even having received one or two doses of vaccine for COVID-19. In the article written by Angel et al. (2021) entitled Association Between Vaccination with BNT162b2 and Incidence of Symptomatic and Asymptomatic SARS-CoV-2 Infections Among Health Care Workers, the authors concluded that the effectiveness of the vaccine in symptomatic SARS-CoV-2 infection reduced the incidence of this infection if you compared with unvaccinated health care worker. But the incidence was not reduced in the asymptomatic health care workers, where they found that vaccinated people were more likely to have SARS-CoV-2 if compared to unvaccinated people [1].

This fact could not be different because the author had already studied the energy pattern of her patients since 2010. Since then, changes in the energy type of her patients have been going on. [2].

Since 2015, the energy pattern of her patients has changed in that the vast majority of their patients including babies, children, teenagers, adults and the elderly are all in the same category, regardless of the type of disease they present, that is a very low energy standard in all analyzed patients' chakras' energy centers [3].

In the article written by Huang (2021) entitled Are We Vaccinating Immunocompetent or Immunocompromised People for COVID 19?, the author said that most people who are receiving vaccine today are being classified as immunodepressed due to the low energy found in the patients she studied. These results are due to the influence of electromagnetic radiation that is disseminated all over the world and therefore, she thinks that the sample she took in the study in Brazil, could be a sample representing what may be happening in the world nowadays [2].

In the study written by Callegaro, et al. (2021) entitled Antibody response to SARS-CoV-2 vaccination is extremely vivacious in subjects with previous SARS-CoV-2 infection, the authors are saying that in persons that have previous SARS-CoV-2 infection, the use of single dose of vaccine can result in production of antibodies similar to full vaccine scheduled in naïve persons [4].

In the article written by Bradley et al. (2021) entitled Antibody responses boosted in seropositive healthcare workers after single dose of SARSCoV-2 mRNA vaccine, the authors are saying that after single dose of Pfizer SARS-CoV-2 vaccine, the persons who had prior SARS-CoV-2 infections will have higher antibodies levels than persons that had no history of previous infection [5].

In the article written by Hellerstein (2020) entitled What are the roles of antibodies versus a durable, high quality $\mathrm{T}$-cell response in protective immunity against SARS-CoV-2, the author is saying that there are concerns about if the antibodies response means that they can give protective immunity. According to the author, antibodies response is a poor marker of prior coronavirus infection (mainly in mild infections) and 
strong antibody response is related to more severe clinical evolution diseases. When there is $\mathrm{T}$ cell response, this means less clinical severe disease. It is still unclear whether the antibodies production is pathogenic or protective in infections by coronavirus [6].

In the article written by Fergie and Srivastava (2021) entitled Immunity to SARS-CoV-2: Lessons Learned, the authors are saying that their knowledge about the durability and extend of immunity and the spectrum of vaccine coverage against SARS-CoV-2 variants still evolving [7].

In the article written by Stiepan (Mayo Clinic-2021) entitled COVID-19 infection among vaccinated people: What you need to know, the author is saying that vaccinated people can still spread COVID-19. In this same article, in the interview with Dr. John O'Horo (that is the infectious diseases specialist in Mayo clinic) he is saying that people should know about the transmission and infections of COVID-19 among people who have been vaccinated for COVID-19. In this article, he is saying that fully vaccinated people still have the potential to transmit COVID-19 to others. The potential of reduction of transmission it is not reduced to allow unmasking in all situations (because people usually understand that the potential of reduction of transmission with vaccination). They are recommending that people still continue using masks even after vaccination, to protect those who are at high risks of transmission, even from vaccinating people. They are also saying that even people that are vaccinated have high levels of virus in the nose and in their throat, even asymptomatic. They affirm that many types of vaccines can prevent illness but do not necessary prevent the transmission of that illness to others [8].

In the article written by Gobbi., et al. (2021) entitled Antibody Response to the BNT162b2 mRNA COVID-19 Vaccine in Subjects with Prior SARS-CoV-2 Infection, they are saying that antibody levels progressively decrease after SARS-CoV-2 infection, that are persistence of immune memory for months and in naturally contracted SARS-CoV-2 infection are expected a more rapidly and sustained response to COVID-19 vaccines than in naïve people [9].

In the article written by Poland et al. (2020) entitled SARS-CoV-2 immunity: review and applications to phase 3 vaccine candidates, they are saying that it is unknown the immunological correlation of protection against COVID-19 and the role of the antibodies specific to SARS-CoV2 and the production of T-cells in elimination the infection. All this information is not identified yet in humans [10].

In the article written by Hacisuleyman et al (2021) entitled Vaccine Breakthrough Infections with SARS-CoV-2 Variants, they are saying that there is a potential risk of COVID-19 disease after vaccination with variant virus and it is important to continue the preventive and diagnosis of infection by variants in vaccinated people [11].

In the article written by Zhou and Wang (2021) entitled Fast-spreading SARS-CoV-2 variants: challenges to and new design strategies of COVID-19 vaccines, they are saying that SARS-CoV-2 replication is accompanying by high mutation rate, that could make the virus more contagious and difficult to eliminate and had been dominated worldwide [12].

In the article written by Huang (2021) entitled Is SARS-CoV- 2 Strong or Our Body Is Weak? the author is demonstrating that patients today are weak in energy and in immunity, as she demonstrated in the article Are We Vaccinating Immunocompetent or Immunocompromised People for COVID 19? In all these articles, the author is showing that the type of population that we have nowadays is different from the type of population that exists 7 years ago, due to lack of energy in the internal massive organs, that is responsible for the production of diverse energy pattern to maintain health and the author is considering that the majority of the population nowadays is considered immune deficient and not immune competent $[2,13]$.

This problem is not discussed in medical faculties and in many scientific articles about this subject because it is in the energy level and this cannot be seeing by the naked eyes. But as said by Albert Einstein (1879 - 1955), the major greatest physics is this world and also said by Capra (1975) in his book Tao of Physics, they both are saying that all the things are made by energy, even all the things that we can see my naked eyes is made by energy. So, we can conclude that human being is also made by energy and it is this part that is affected nowadays, that is not recognized by the scientific community, due to some implementations in the past, after Flexner report, that only consider scientific only what you can proof by laboratorial or radiological level. This made the Western diagnosis were late because all diseases come from energy imbalances (that is characterized by alterations in the phase one to three of the evolution from health to disease) [14-16].

To improve vital energy of all organs that are weak nowadays, as demonstrated by Huang (2021) in the article Energy Alterations and Chakras' Energy Deficiencies and Propensity to SARS-CoV-2 Infection, the author is demonstrating that we can increase the energy of the internal massive organs, to increase the energy of the Spleen-pancreas ( that is deficient in $97 \%$ of the patients studied by the author and it is responsible for the production of blood and white blood cells ) and to increase the Zheng-Qi of the patient ( that is the energy to protect the body against the invasion of the external pathogenic factors ( and SARS-CoV-2 is considered invasion of external pathogenic factor) $[2,3]$.

To improve the immunity of those people who have already taken the COVID-19 vaccine, because nowadays, the people that is receiving vaccination still have poor immune system to produce antibody and poor immune defense against the protection of many other infectious and noninfectious diseases. That is why, the author is showing the necessity of treating this deficiency, even the people received vaccination, to improve the energy of the 5 massive internal organs (Liver, Heart, Spleen, Lung and Kidney), as Huang (2021) showed in article Energy Alterations and Chakras' Energy Deficiencies and Propensity to SARS-CoV-2 Infection and in the article Are We Vaccinating Immunocompetent or Immunocompromised People for COVID 19? Using these kind of tools, the people will be more protected due to the defense and the energy that is responsible for the production of white Blood and production of $\mathrm{T}$ and B cells inside the body, will be working more efficiently, because you will give the energy that is lacking nowadays due to the influences of the electromagnetic waves, that is affecting our body, leading to this immune suppression $[2,3]$.

In Chine, when some people have internal massive organ deficient in energy, they usually use the Chinese herbs to tone the energy of one specific organ. As the author lives in Brazil, she has some problems in prescription and to have this kind of herbs to prescribe for her patients and she started to learn homeopathy in 2015. During her course, she noticed that homeopathy is a very efficient medication to be used in a diverse situations nowadays and she began to write another theory linking homeopathy (that was created by Hahnemann and exists for 242 years since the first publication) with traditional Chinese medicines reasoning (that exists for more than 5000 years). All her articles were following Hippocrates thoughts that said that it is important to consider other ancient medical traditions prior to the knowledge we have nowadays. So, Huang (2020) created a theory entitled Constitutional Homeopathy of the Five Elements based on Traditional Chinese Medicine, that is the way that medical doctors can use to treat these lack of energy, responsible for the formation of the majority of chronic diseases, including the formation of this pandemic of COVID-19, due to this reduced immune system in the whole population in this globe [17]. 
To tone the Liver (first chakra) or other internal organ, the physician needs to tone all the internal organs that are weak in energy also, because all organs are interconnected by energy flow, different from the Western perspective, that thinks that all organs are independently and do not exert influences in other organs. To analyze which organ is low in energy, the author uses the radiesthesia procedure, measuring all the chakras from all her patients. If we measure the chakras' energy centers of the patient, we will be measuring the energy of the five massive organs, as showed in most articles written by the author such as in the article Why Are Diabetic Patients Still Having Hyperglycemia despite Diet Regulation, Antiglycemic Medication and Insulin? [14].

To tone the Heart (third chakra) the physician need to use Sulphur. To tone the Spleen (fifth chakra), it can use Calcarea carbonica. To tone the Lungs (fourth chakra) the doctor can use Silicea and to tone the Kidney (second chakra) the physician can use Natrum muriaticum. All this information is written in diverse publication of the author, as in the article Constitutional Homeopathy of the Five Elements based on Traditional Chinese Medicine [17].

In addition to the use of these medications, the author recommends the use of nosode of SARS-CoV-2. This is a homeopathic medicine, that is made by secretion of patients who had COVID-19, following Hahnemann reasoning that said that, if you give any substance for a health person, he can have this disease. But if you dilute this same substance in a very infinitesimal mode, this same substance can be the medication to treat this infection. Depends on, if the physician will give the medication for prevention or for treatment, the potency of the medication will differ, These information were published by Huang (2021) in the article entitled Chakras Energies Deficiencies as the Cause of Fatigue Post Sars-Cov-2 Infection Patients Treatment [18].

Homeopathy was discovered by the German doctor Hahnemann (17551843) and as soon as it was created, it received many barriers to its application since inside it, there are no signs of matter but only energy. But according to Arndt Schultz Law, created by two German researchers, they said that highly diluted medications can improve the vital energy, and highly concentrated medications can reduce the vital energy. That is why homeopathy is considered the medication of choice nowadays; to treat all kinds of diseases, as showed in the article Is the population the same as in the past? [19,20].

In the book written by Manning and Vanrenen (1993) entitled Bioenergetic Medicines East and West: Acupuncture and Homeopathy, the authors are saying that homeopathy works as bioenergy as an effect of acupuncture [21].

Therefore, the author recommends that even if the patient had been vaccinated to prevent COVID-19, patients should improve their internal energy and improve their immunity, which nowadays is impaired due to the constant exposure to electromagnetic energy, by which they are being affected and which nowadays Western medicine still cannot identify because after the implementation of Flexner's report in 1913, changing the entire curriculum of medical schools in the three largest continents on the planet, they understand that only what is scientific is what they can see with their naked eyes and what can be proven in laboratory and radiological examinations. But the part of the energy that makes up the body, that is considered unscientific nowadays but it is the part that is of extreme importance to understanding of the causes of reactions, complications and deaths in this COVID-19 pandemic scenario. This lack of energy can explain the reduced response of the patients to the vaccination, as showed in the article written by Jevanathan et al (2020) entitled Immunological considerations for COVID-19 vaccine strategies, where the authors are saying that the neutralizing antibody production in asymptomatic individuals decrease faster and it is small than in symptomatic individuals. In this article, they are saying that quality and longevity of vaccine induced protection is unavailable [22].

Therefore, the understanding of the human being as a holistic being made of energy and matter, is important for understanding how to prevent the acquisition of COVID-19 even if today they have already been vaccinated by the disease, because the energy of quite entire population in this world is affected by the exposition to electromagnetic waves, reducing the energy of the five massive organs, that are responsible for the production of Blood cells, that is responsible for the antibody production . As said by Huang (2021) in the article entitled Are the Vaccines the Only Solution to Control COVID-19 Pandemic? the author is saying that due to this lack of energy, leading to the state of immune deficiency, there is the necessity of treating this part of deficiency that still not studied by Western medicine, that is the real cause of the persistent infection, either with vaccination, caused in the infection of other strains of SARS-CoV-2. If we increase the energy or immunity of all population, we will protect the people against all infections, including all SARS-COV-2 strains that is existed nowadays [23].

As said by Hippocrates, we need to treat the patient and not the disease. According to him, it is more important to know which kind of person has the disease than to know which disease the person has [24].

\section{References}

1. Angel et al. (2021). Association between Vaccination with BNT162b2 and Incidence of Symptomatic and Asymptomatic SARS-CoV-2 Infections among Health Care Workers. JAMA. 325(24):2457-2465.

2. Huang WL. (2021). Are We Vaccinating Immunocompetent or Immunocompromised People for COVID 19? J Vaccines Res Vaccine.

3. Huang Wei Ling. (2021). "Energy Alterations and Chakras' Energy Deficiencies and Propensity to SARS-CoV-2 Infection". Acta Scientific Microbiology. 4(4): 167-196.

4. Callegaro A, et al. (2021). Antibody response to SARS-CoV-2 vaccination is extremely vivacious in subjects with previous SARS-CoV-2 infection. J Med Virol. 93(7):4612-4615.

5. Bradley T, et al. (2021). Antibody responses boosted in seropositive healthcare workers after single dose of SARSCoV-2 mRNA vaccine. medRxiv.

6. Hellerstein M. (2020). What are the roles of antibodies versus a durable, high quality $\mathrm{T}$-cell response in protective immunity against SARS-CoV-2? Vaccine X.

7. Fergie J; Srivastava A. (2021). Immunity to SARS-CoV-2: Lessons Learned. Front Immunol.

8. COVID-19 infection among vaccinated people What you need to know. Mayo Clinic.

9. Gobbi et al. (2021). Antibody Response to the BNT162b2 mRNA COVID-19 Vaccine in Subjects with Prior SARS-CoV2 Infection. Viruses. 13(3):422.

10. Poland G, et al. (2020). SARS-CoV-2 immunity: review and applications to phase 3 vaccine candidates. Lancet. 396(10262):1595-1606.

11. Hacisuleyman et al. (2021). Vaccine Breakthrough Infections with SARS-CoV-2 Variants. N Engl J Med. 384(23):22122218.

12. Zhou and Wang. (2021). Fast-spreading SARS-CoV-2 variants: challenges to and new design strategies of COVID-19 vaccines. Signal Transduct Target Ther. 6: 226.

13. Ling HW. (2021). Is SARS-CoV- 2 Strong or Our Body Is Weak? J Dent Oral Epidemiol. 1(1).

14. Huang Wei Ling. (2019). Why Are Diabetic Patients Still Having Hyperglycemia Despite Diet Regulation, Antiglycemic 
Medication and Insulin? Int J Diabetes Metab Disord. 4(2):114.

15. Capra, Fritjof. (1975). The Tao of Physics: an Exploration of the Parallels between Modern Physics and Eastern Mysticism. Berkeley: [New York]:Shambhala ; distributed in the U.S. by Random House.

16. Huang Wei Ling. "What Flexner Report did to Our Medicine After 100 Years of Implantation?". Acta Scientific Gastrointestinal Disorders 4.8 (2021): 01-04.

17. Huang Wei Ling. (2020). Constitutional Homeopathy of Five Elements Based on Traditional Chinese Medicine. Acta Scientific Medical Sciences. 4(7):57-69.

18. Huang WL. (2021). "Chakras' Energy Deficiencies as the Cause of Fatigue Post SARS-CoV-2 Infection Patients Treatment". Acta Scientific Microbiology. 4(6): 91-108.
19. LEESER O. (1953). Support of homeopathy by the ArndtSchulz law. National Center for Biotechnology Information.

20. Huang Wei Ling. "Is the Population in the World the Same as in the Past?". Acta Scientific Clinical Case Reports 2.6 (2021).

21. Clark Manning, Louis Vanrenen. (1993). Bioenergetic Medicines East and West: Acupuncture and Homeopathy Paperback.

22. Jevanathan et al. (2020). Immunological considerations for COVID-19 vaccine strategies. Nat Rev Immunol. 20(10):615632.

23. Huang Wei Ling. (2021). Are the Vaccines the Only Solution to Control COVID-19 Pandemic?. Vaccines Vacccin. 6(1): 000152.

24. Craik E. (2014). "The "Hippocratic" Corpus: Content and Context". Routledge: 344.
This work is licensed under Creative Commons Attribution 4.0 License

\section{To Submit Your Article Click Here: Submit Manuscript}

DOI: $10.31579 / 2692-9406 / 081$
Ready to submit your research? Choose Auctores and benefit from:

$>$ fast, convenient online submission

$>$ rigorous peer review by experienced research in your field

$>$ rapid publication on acceptance

$>$ authors retain copyrights

$>$ unique DOI for all articles

$>$ immediate, unrestricted online access

At Auctores, research is always in progress.

Learn more www.auctoresonline.org/journals/biomedical-research-andclinical-reviews- 